\title{
Expression profile of tRNA-derived fragments and their potential roles in human varicose veins
}

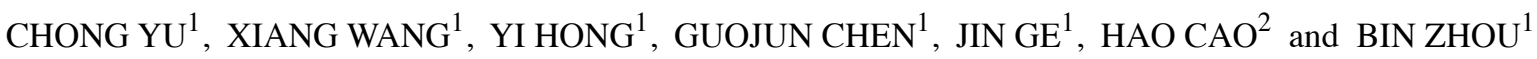 \\ Departments of ${ }^{1}$ Vascular Surgery and ${ }^{2}$ Cardiovascular Surgery, \\ Shanghai East Hospital Affiliated to Tongji University School of Medicine, Shanghai 200120, P.R. China
}

Received December 23, 2018; Accepted June 19, 2019

DOI: $10.3892 / \mathrm{mmr} .2019 .10544$

\begin{abstract}
Varicose veins (VVs) is a common disease presenting with chronic venous insufficiency. tRNA-derived fragments (tRFs) are associated with a variety of pathological conditions. However, the functions of tRFs in VVs have not been elucidated to date. The present study aimed to identify the key tRFs and investigate their potential roles in VVs. Small RNA sequencing (RNA-seq) was performed to investigate the expression of tRFs in tissues of patients with VVs and their matched adjacent normal veins tissues (ANVs). Reverse transcription-quantitative PCR (RT-qPCR) was used to confirm the differential expression of tRFs. A total of 13,789 tRFs were identified by small RNA-seq, including 45 differentially expressed tRFs (DETs), which comprised 14 upregulated and 31 downregulated tRFs in VV tissues compared with ANVs. In addition, DETs were mainly involved in the function of epidermal growth factor receptor and vascular endothelial growth factor receptor signaling pathways in VVs. Kyoto Encyclopedia of Genes and Genomes (KEGG) analysis revealed that the target genes of DETs were predominantly involved in Wnt and mitogen-activated protein kinase (MAPK) signaling pathways, as well as calcium signaling. Additionally, two upregulated tRFs (tRF-36-F900BY4D84KRIME and tRF-23-87R8WP9IY) and one downregulated tRF (tRF-40-86J8WPMN1E8Y7Z2R) were further validated by RT-qPCR, and a signaling pathway regulation network of their target genes confirmed their involvement in the calcium, Wnt and MAPK signaling pathways. The results of the present study identified three DETs (tRF-36-F900BY4D84KRIME,
\end{abstract}

Correspondence to: Dr Bin Zhou, Department of Vascular Surgery, Shanghai East Hospital Affiliated to Tongji University School of Medicine, 150 Jimo Road, Pudong, Shanghai 200120, P.R. China

E-mail: mcdropin@126.com

Abbreviations: VVs, varicose veins; tRFs, tRNA-derived fragments; ANVs, matched adjacent normal vein tissues; DETs, differentially expressed tRFs; KEGG Kyoto Encyclopedia of Genes and Genomes

Key words: tRNA-derived fragments, varicose veins, small RNA-sequencing, signaling pathways
tRF-23-87R8WP9IY and tRF-40-86J8WPMN1E8Y7Z2R), which may have crucial roles in the occurrence and progression of VVs by regulating Wnt and MAPK signaling, as well as calcium signaling. The present results may provide a basis for further investigation of the functional roles of tRFs in VVs.

\section{Introduction}

Varicose veins (VVs) is one of the most common diseases with chronic venous insufficiency that affects $\sim 73 \%$ females and $56 \%$ males worldwide (1). A number of factors are involved in the occurrence of VVs, such as constrictive underclothes, standing for long periods of time and suffering from diabetes mellitus and/or hypertension, which can lead to blood accumulation in the lower extremities of the body (2). Recent advances have suggested that reflux induced by venous valve dysfunction (3), intimal hyperplasia, alterations in the extracellular matrix (ECM), abnormal glucose metabolism, local inflammation, local hypoxia, endothelial cell injury, smooth muscle cell proliferation and apoptosis, and increased vein wall tension (4-6) may be associated with the mechanistic changes resulting in the development and progression of VVs. However, these advancements account for a limited number of putative mechanisms and thus, it is important to further investigate the molecular mechanisms involved in the pathogenesis of VVs and improve the existing therapeutic strategies.

tRNA-derived fragments (tRFs), which are 15-50 nucleotides long, are members of the small RNA family that are derived as unique sequences from mature tRNAs or precursor tRNAs. With the advances in next-generation sequencing and bioinformatics tools, tRFs have been successfully detected in eukaryotic cells and demonstrated to participate in various pathological processes $(7,8)$. tRFs serve key roles in physiological processes, such as regulating mRNA stability $(9,10)$, inhibiting translation initiation and elongation $(11,12)$, regulating ribosome biogenesis (13), functioning as an epigenetic factor (14), regulating RNA reverse transcription as a guide RNA (15), preventing apoptosis by binding to cytochrome c (16), immune regulation (17) and degradation of target RNAs, such as mRNAs and microRNAs (miRNAs) (18-20). Several studies have reported that tRFs are involved in a number of diseases, including cancer $(21,22)$, neurodegenerative diseases (12), acquired metabolic diseases (23) and infectious diseases (24). Additionally, tRFs have been demonstrated to 
function as biomarkers in the diagnosis of these diseases (25). However, the potential molecular mechanism of tRFs in the occurrence and progression of VVs remains poorly understood.

In the present study, the key tRFs and their potential molecular mechanisms in VVs were investigated, the differentially expressed tRFs (DETs) in VVs were identified using small RNA sequencing (RNA-seq), and the potential functional roles of these tRFs were further analyzed using bioinformatic tools.

\section{Materials and methods}

Patients and samples. This study was approved by the Ethics Committee of Shanghai East Hospital (Shanghai, China) and all patients enrolled in the present study provided written informed consent. All experiments were performed following the Code of Ethics of the World Medical Association. Primary VVs were obtained from 24 patients who underwent open surgery or endovenous laser treatment. Phlebectomy was performed to obtain vascular tissue exhibiting signs of VVs and matched adjacent normal vascular tissue (ANV) from patients undergoing surgery at Shanghai East Hospital between July 2017 and January 2018. The clinical information of patients with VVs is presented in Table I. The vascular tissues of patients with VV and the matched ANVs were stored at $-80^{\circ} \mathrm{C}$ until experimental use.

Library preparation and RNA-seq of small RNA. Samples used for sequencing were selected randomly. Total RNA from VVs and matched ANVs $(\mathrm{N}=4)$ was extracted from the vascular tissue. Small RNAs (135-170 bp) were isolated using a QIAquick gel extraction kit (Qiagen, Inc.). A miRNA library was constructed by Ying Biotech from the isolated purified small RNA using NEBNext ${ }^{\circledR}$ Multiplex Small RNA Library Prep kit (cat. no. E7560S; New England Biolabs, Inc.), followed by analysis of the small RNA-seq data. Briefly, miRNA was bound with $3^{\prime}$ and 5'-adapters, and cDNA constructs were created by RT-PCR. The miRNA library was submitted for deep sequencing on Illumina $\mathrm{HiSeq}^{\mathrm{TM}} 2500$ platform (Illumina Inc.).

Bioinformatics analysis. The raw sequencing data was used to filter low quality and short reads ( $<15$ nucleotides). To identify known small RNAs, all small RNA clean reads were aligned to the miRBase database (http://www.mirbase.org). Unmapped reads with miRBase database were aligned to Genomic tRNA database (http://gtrnadb.ucsc.edu) prior to alignment to tRFdb (http://genome.bioch.virginia.edu/trfdb) and MintBase (http://cm.jefferson.edu/MINTbase/). Eventually, tRFs were obtained by mapping these databases. Unmapped reads from the Genomic tRNA database were aligned to other corresponding databases of small RNAs (piRNA, small nuclear RNA, small nucleolar RNA and other small RNAs). The tRFs with significantly different expression levels between tissues of patients with VVs and matched ANVs were selected based on statistical values of $\log _{2}$ fold change $\left(\log _{2} \mathrm{FC}\right)>1$ or $<-1$ and false discovery rate (FDR) $<0.05$ using EBSeq (https://www.biostat.wisc.edu/ kendzior/EBSEQ/) based on negative binomial distributions. mRNAs and target genes of the differently expressed tRFs were predicted using miRanda (http://www.microrna.org/microrna/home.do) and RNAhybrid
Table I. Clinicopathological characteristics of patients with varicose veins.

\begin{tabular}{lc} 
Characteristic & Patients $(\mathrm{n}=24)$ \\
\hline Age (years) & $64.2 \pm 9.9$ \\
Female & 13 \\
Male & 11 \\
Hypertension & 13 \\
Diabetes Mellitus & 1 \\
Left limb operation & 13 \\
Right limb operation & 11 \\
CEAP (grade) & $3.7 \pm 0.7$
\end{tabular}

Continuous data are presented as mean \pm standard deviation and categorical data are presented as counts. CEAP, clinical, etiological, anatomical and pathological elements classification system.

(https://bibiserv.cebitec.uni-bielefeld.de/rnahybrid). The intersection of miRanda (score $>150$; energy <-20) and RNAhybrid (energy <-25) was revealed to be significant. The functions and pathways of the majority of the target genes were classified using the Gene Ontology (GO; http://www.geneontology.org/), and Kyoto Encyclopedia of Genes and Genomes (KEGG; http://www.genome.jp/kegg) databases. GO terms were classified as biological processes (BP), molecular functions (MF) and cell components (CC). In addition, a network between tRFs, target genes and pathways was performed using cytoscape 3.6.1 (https://cytoscape.org) (26).

$\log _{2}(\mathrm{FC})$ was used to represent the signal difference between the experimental group and the control group calculated by the logarithm to base 2, and FDR was used to determine the multiple hypothetical testing error misjudgment rates. Lower FDR represented fewer errors.

$R N A$ extraction and reverse transcription-quantitative $P C R$ $(R T-q P C R)$. Total RNA was extracted from vascular tissues exhibiting VVs and the matched ANVs of 20 patients using TRIzol $^{\circledR}$ reagent (Invitrogen; Thermo Fisher Scientific, Inc.) according to the manufacturer's protocol. Quality and concentration of RNA was assessed using NanoDrop ND1000 spectrophotometer (Thermo Fisher Scientific, Inc.). Isolated RNA was reverse transcribed into cDNA using RevertAid ${ }^{\mathrm{TM}}$ Fist Strand cDNA Synthesis kit (cat. no. K1622; Thermo Fisher Scientific, Inc.) according to the manufacturer's protocol.qPCR was performed using SYBR Green PCR kit (Qiagen, Inc.) and detected on an ABI Q6 detection system (Applied Biosystems; Thermo Fisher Scientific, Inc.). The thermocycling conditions were as follow: $95^{\circ} \mathrm{C}$ for $10 \mathrm{~min}$ followed by 45 cycles of $95^{\circ} \mathrm{C}$ for $15 \mathrm{sec}, 60^{\circ} \mathrm{C}$ for $1 \mathrm{~min}$; the melting conditions were $95^{\circ} \mathrm{C}$ for $10 \mathrm{sec}, 60^{\circ} \mathrm{C}$ for $1 \mathrm{~min}, 95^{\circ} \mathrm{C}$ for $15 \mathrm{sec}$ for one cycle. U6 was used as the internal control, and relative quantification and calculations were done using the $2^{-\Delta \Delta \mathrm{Cq}}$ method (27). Primers were designed by Ying Biotech. The primer sequences are listed in Table II.

Statistical analysis. Statistical analysis was performed using SPSS version 12.0 statistical software (SPSS, Inc.) and the data 
Table II. Primer sequences.

Name

U6-F

U6-R

tRF-36-F900BY4D84KRIMEF

tRF-36-F900BY4D84KRIMER

tRF-23-87R8WP9IYF

tRF-23-87R8WP9IYR

tRF-40-86J8WPMN1E8Y7Z2RF

tRF-40-86J8WPMN1E8Y7Z2RR
Primer sequences

F, forward; R, reverse; tRF, tRNA-derived fragments.
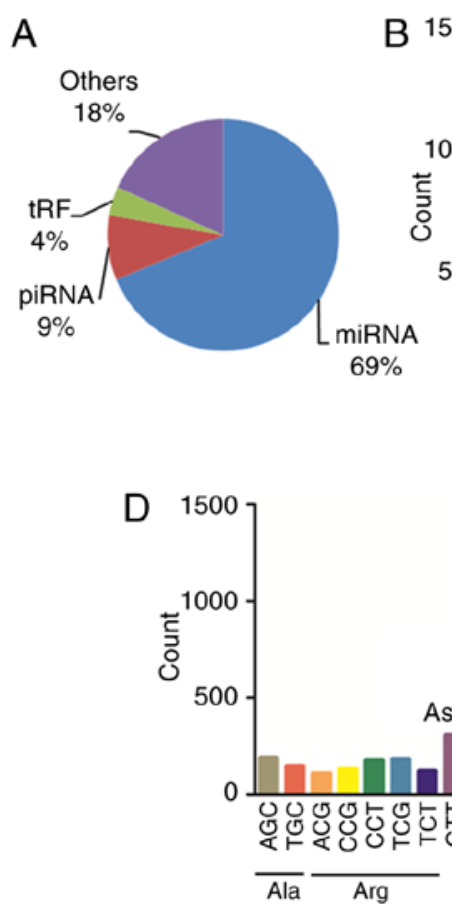

5'-CGATACAGAGAAGATTAGCATGGC-3'

5'-AACGCTTCACGAATTTGCGT-3'

5'-ACATGGTCTAGCGGTTAGGATTC-3'

5'-AGTGCGTGTCGTGGAGTCG-3'

5'-CGCAGTCCCTGGTGGTCTAGT-3'

5'-AGTGCGTGTCGTGGAGTCG-3'

5'-AGCTCACAAGAACTGCTAACTCATG-3'

5'-AGTGCGTGTCGTGGAGTCG-3'

Figure 1. Characteristics of tRFs in human varicose veins. (A) The percentage of different types of small RNAs. Blue, miRNA; red, piRNA; green, tRF; purple, other small RNAs. (B) Length-wise distribution of tRFs. (C) Quantitative analysis of different subtypes of tRFs. (D) The numbers of tRF genes corresponding to different amino acids and anticodons. Each amino acid is represented by a different color. tRFs, tRNA-derived fragments; miRNA, microRNA; piRNA, piwi-interacting RNA; i-tRF, internal tRF.

were visualized using GraphPad Prism 5.0 (GraphPad Software, Inc.). The RT-qPCR data were presented as the mean \pm standard deviation and analyzed using paired Student's t-test. $\mathrm{P}<0.05$ was considered to indicate a statistically significant difference.

\section{Results}

Conventional characteristics of tRFs. To study the potential function of tRFs in VVs, small RNA-seq was performed on the resected VV and matched ANV tissues from patients with VVs $(\mathrm{N}=4)$. To further elucidate the common characteristics of tRFs in human VVs, a detailed preliminary analysis of the sequencing data was performed. The results demonstrated that tRFs accounted for $4 \%$ of the total small RNAs (Fig. 1A). The length of tRFs was observed to be 15-50 nucleotides (Fig. 1B).
A total of 13,789 tRFs were identified, which comprised the following subtypes: 3'-half $(\mathrm{N}=226), 5$ '-half $(\mathrm{N}=293)$, internal tRF (i-tRF; N=8,908), tRF-1 (tRFs derived from the 3 'trailer fragment of precursor tRNAs; $N=29$ ), tRF-3 (tRFs derived from the extreme 3 'ends of mature tRNAs; $N=2,831$ ) and tRF-5 (tRFs from the extreme 5'ends of mature tRNAs; $N=1,502$ ), as presented in Fig. 1C. The analysis revealed that most tRFs subtypes were transcribed from i-tRF. Fig. 1D shows the that the tRFs derived from tRNA(Glu) were the most abundant, followed by that from tRNA(Gly) and tRNA(Lys).

Identification of DETs in VVs. To increase the reliability of differential expression detected from the expression profile between $\mathrm{VV}$ and matched ANV tissues, tRFs with low signal intensities or those which were not consistently expressed in all the tissues 
Table III. Top 10 downregulated and upregulated tRNA fragments.

A, Top 10 downregulated tRFs

\begin{tabular}{lccrr}
\hline Acc ID & Subtype & Anticodons & Length (bp) & FC \\
\hline tRF-37-86J8WPMN1E8Y7Z2 & tRF-5 & GluTTC & 37 & -1.723 \\
tRF-36-MIF91SS2P4FINOE & i-tRF & LysCTT & 36 & -1.715 \\
tRF-35-86J8WPMN1E8Y7Z & 5 '-half & GluTTC & 35 & -1.712 \\
tRF-37-MIF91SS2P4FINO5 & i-tRF & LysCTT & 37 & -1.678 \\
tRF-40-86J8WPMN1E8Y7Z2R & tRF-5 & GluTTC & $\mathbf{4 0}$ & $\mathbf{- 1 . 6 6 9}$ \\
tRF-34-PS5P4PW3FJHPEQ & 5'-half & LysTT & 34 & -1.571 \\
tRF-38-HPDE8P7SER9I69DV & tRF-3 & ArgACG & 38 & -1.542 \\
tRF-18-RPM83004 & tRF-5 & LeuTAG, LeuAAG & 18 & -1.04 \\
tRF-17-RPM830P & tRF-5 & LeuTAG, LeuAAG & 17 & 0.049 \\
tRF-17-RPM830K & tRF-5 & LeuTAG, LeuAAG & 17 & -1.424 \\
\end{tabular}

B, Top 10 upregulated tRFs

\begin{tabular}{|c|c|c|c|c|c|}
\hline Acc ID & Subtype & Anticodons & Length (bp) & $\mathrm{FC}$ & P-value \\
\hline tRF-21-2MK8J7K1B & tRF-3 & AlaTGC, AlaCGC & 21 & 1.407 & 0.040 \\
\hline tRF-31-ROD8NOX0JYOYE & 5'-half & TyrGTA & 31 & 1.570 & 0.039 \\
\hline tRF-18-PNR8YP04 & tRF-5 & GlyCCC, GlyGCC & 18 & 1.659 & 0.034 \\
\hline tRF-23-87R8WP9IY & tRF-5 & GluTTC & 23 & 1.693 & 0.047 \\
\hline tRF-33-LBINRXURYRSIV & tRF-3 & MetCAT & 33 & 1.722 & 0.031 \\
\hline tRF-41-Z4D7OOJ0QXL13XZ5D & $\mathrm{i}-\mathrm{tRF}$ & HisGTG & 41 & 2.021 & 0.028 \\
\hline tRF-25-690M0RHB26 & tRF-3 & PheGAA & 25 & 2.317 & 0.049 \\
\hline tRF-30-DKNPNOYB7JXN & 5'-half & AlaTGC & 30 & 2.431 & 0.031 \\
\hline tRF-30-PW5SVP9N15WV & tRF-5 & HisGTG & 30 & 2.497 & 0.018 \\
\hline tRF-36-F900BY4D84KRIME & i-tRF & SerGCT & 36 & 3.575 & 0.008 \\
\hline
\end{tabular}

Bold font denotes the three tRFs that were validated in the present study by reverse transcription-quantitative PCR. Acc ID, the name of the tRF in the database; FC, fold change; tRF, tRNA fragment.

were filtered out. From the remaining signals, tRFs that exhibited at least a 2-fold expression change and $\mathrm{P}<0.05$ were considered differentially expressed and were selected for further analysis. Overall, the small RNA-seq analysis revealed 45 DETs in VVs compared with the matched ANV tissues (Fig. 2A). Of these, 14 tRFs were identified to be significantly upregulated and 31 tRFs were significantly downregulated (Fig. 2B). A detailed list of the 45 DETs identified in the present study is presented in Table III. The results suggested that the expression pattern of tRFs in VVs was distinctly different compared with that in ANVs.

GO and KEGG pathway enrichment analysis of target genes of DETs. tRFs regulate gene expression and degradation of target RNAs, such as mRNA and miRNA (18-20). To further explore the potential functions of tRFs in VVs, the target genes of DETs were theoretically predicted using miRanda and RNAhybrid. The results demonstrated that 8,114 genes were associated with the 45 DETs in VVs, which involve 22,405 binding sites (Fig. 3A). To further understand the biological functions of these target genes, GO and KEGG pathway enrichment analysis was performed. In the GO analysis, the enrichment score was calculated as $-\log 10$ ( $\mathrm{P}$-value) $(\mathrm{P}<0.05)$. A total of 656 GO terms were enriched in GO-BP. The dataset included BPs such as 'epidermal growth factor receptor signaling pathway' (GO: 0007173; $\mathrm{P}=2.975 \times 10^{-9}$ ) and 'vascular endothelial growth factor (VEGF) receptor signaling pathway' (GO: 0048010; $\left.\mathrm{P}=5.593 \times 10^{-9}\right)$, which are associated with vascular disease (Fig. 3B) $(28,29)$. In GO-MF, 208 terms were significantly enriched, which included 'protein binding' (GO: 0005515; $\mathrm{P}=1.848 \times 10^{-21}$ ) and 'transcription factor binding' (GO: 0008134; $\mathrm{P}=2.454 \times 10^{-6}$; Fig. 3C); in GO-CC, 131 terms were significantly enriched, including 'cytoplasm' (GO: 0005737; $\mathrm{P}=1.61 \times 10^{-16}$ ) and 'nucleus' (GO: 0005634; $\mathrm{P}=1.083 \times 10^{-10}$; Fig. 3D). KEGG pathway enrichment analysis revealed that these target genes were significantly enriched in 6,401 signaling pathways, such as 'Wnt signaling pathway' (PATH: 04310; $\mathrm{P}=4.31 \times 10^{-10}$ ), 'calcium signaling pathway' (PATH: 04020; $\mathrm{P}=5.36 \times 10^{-10}$ ) and 'MAPK signaling pathway' (PATH: 04010; $\mathrm{P}=8.395 \times 10^{-8}$; Fig. 3E). These pathways were also associated with vascular disease. Thus, the results suggested that tRFs may serve a role in VVs by regulating Wnt, calcium and MAPK signaling pathways. 


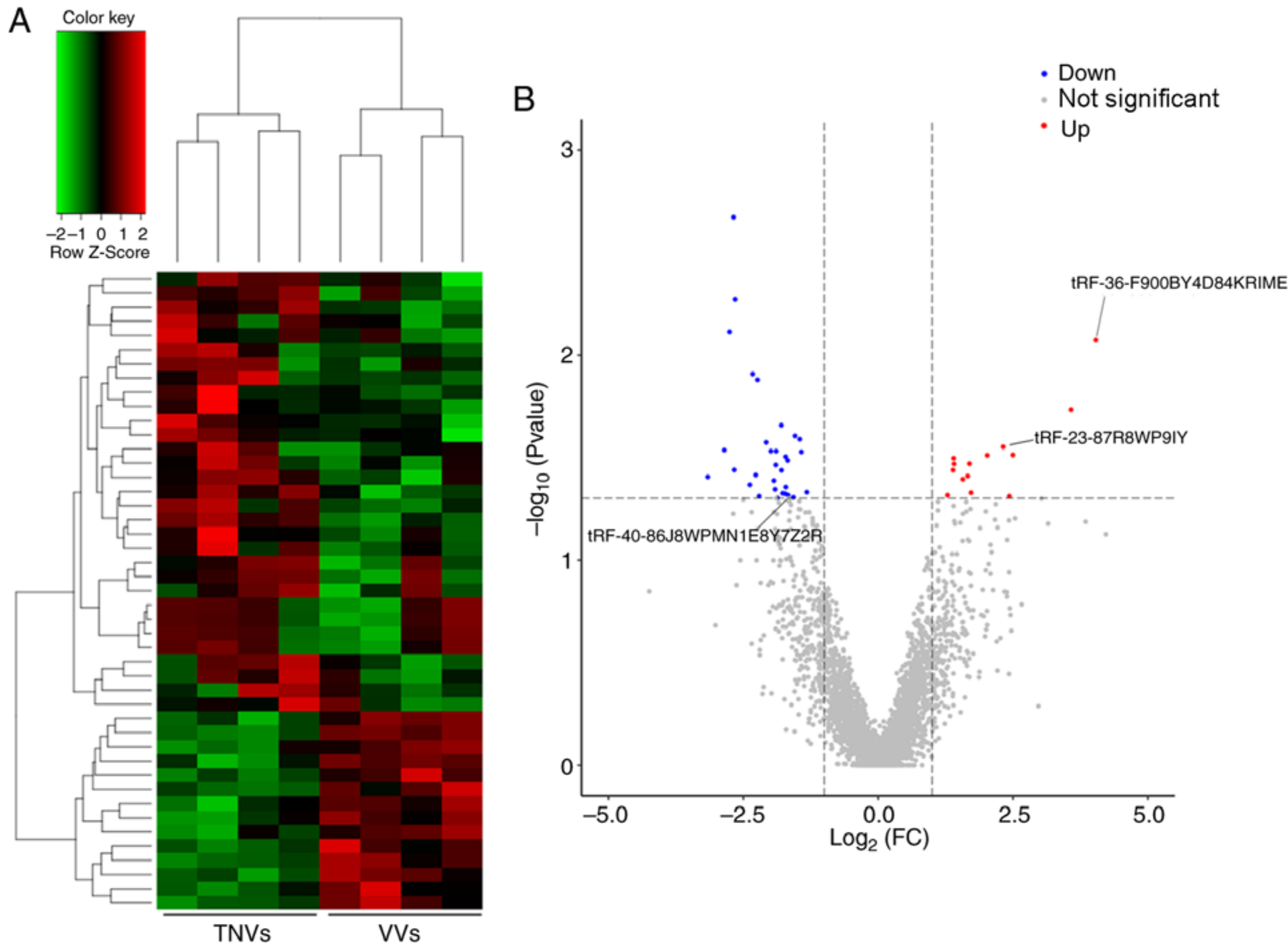

Figure 2. Comparison of DETs between healthy control and VV tissues. (A) Hierarchical cluster analysis of the 45 identified DETs. Red, high relative expression; black, medium relative expression; green, low relative expression. (B) Volcano plot for the tRFs from the RNA-seq analysis. Red, significantly upregulated tRFs; blue, significantly downregulated tRFs; gray, no significant change. DETs, differentially expressed tRFs; VV, varicose vein; ANV, adjacent normal vein; tRFs, tRNA-derived fragments.

Experimental validation of $t R F$ expression using RT-qPCR. A precise list of the DETs identified in the present study based on their comprehensive raw signal intensities, and the final $\mathrm{FC}>2$ and $\mathrm{P}<0.05$ criteria, is provided in Table III. Based on their high abundance and fold changes, three tRFs were shortlisted, which comprised two upregulated tRFs (tRF-36-F900BY4D84KRIME and tRF-23-87R8WP9IY) and one downregulated tRF (tRF-40-86J8WPMN1E8Y7Z2R). To independently confirm the differential expression of these tRFs between $\mathrm{VV}$ and matched ANV samples, RT-qPCR was performed to determine their expression levels $(\mathrm{N}=20)$. The results revealed that in VV tissues, tRF-23-87R8WP9IY and tRF-36-F900BY4D84KRIME were significantly upregulated $(\mathrm{P}=0.001$ and $\mathrm{P}=0.013$, respectively), while tRF-40-86J8WPMN1E8Y7Z2R was significantly downregulated $(\mathrm{P}=0.016)$ compared with matched ANV tissues (Fig. 4). The results were consistent with those of the small RNA-seq analysis, confirming the overall validity of the RNA-seq data, and highlighting the potential gene regulatory roles of the three DETs in VVs.

Signaling pathway regulation network of DETs. To further understand the regulatory mechanism of the three DETs in VVs, a regulation network of the target genes of the shortlisted tRFs was constructed (Fig. 5). The network was integrated and analyzed using Cytoscape. The network demonstrated that the target genes of the three tRFs may be involved in VVs by regulating Wnt, calcium and MAPK signaling pathways, which are associated with vascular diseases (30-32). Moreover, stearoyl-CoA desaturase (SCD), FK506 binding protein 5 (FKBP5) and toll-like receptor 6, which have been shown to be involved in inflammation (33-36), were regulated by the differentially expressed tRFs (tRF-36-F900BY4D84KRIME, tRF-23-87R8WP9IY and tRF-40-86J8WPMN1E8Y7Z2R; Table SI).

Thus, tRF-36-F900BY4D84KRIME, tRF-23-87R8WP9IY and tRF-40-86J8WPMN1E8Y7Z2R may serve important roles in the molecular mechanism of VVs. These findings may help elucidate the potential function of tRFs in the occurrence and progression of VVs.

\section{Discussion}

In the present study, 45 DETs, which included 14 upregulated and 31 downregulated tRFs, were identified to be significantly altered in VVs compared with in ANVs. The target genes of the DETs were significantly enriched in Wnt, calcium and MAPK signaling pathways. In addition, two upregulated tRFs and one downregulated tRF were shortlisted, which may regulate Wnt, calcium and MAPK signaling pathways. The results of the present study suggest that tRFs may serve significant roles in 


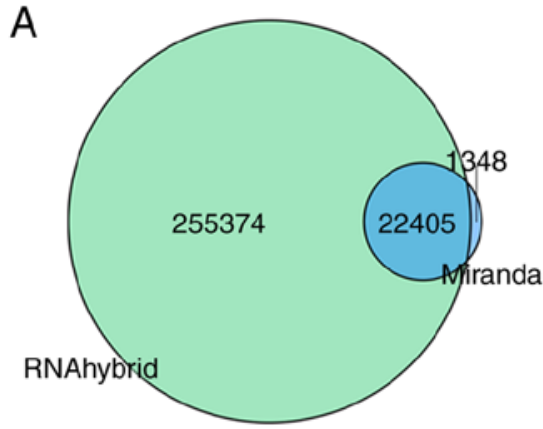

B

GO-Analysis_BP

Protein dephosphorylation-

Multicellular organismal development-

Positive regulation of MAPK cascadeNegative regulation of transcription from RNA polymerase II promoterFibroblast growth factor receptor signaling pathway-

Cell adhesionTransport

Intracellular signal transductionInsulin receptor signaling pathwayPhosphorylation-

Positive regulation of transcription from RNA polymerase II promoterHomophilic cell adhesion via plasma membrane adhesion molecules Vescular endothelial growth factor receptor signaling pathwayEpidermal growth factor receptor signaling pathwayNeurotrophin TRK receptor signaling pathway ceptor signaling pathway Axon guidanceNervous system development Transcription DNA-templated Regulation of transcription DNA-templated

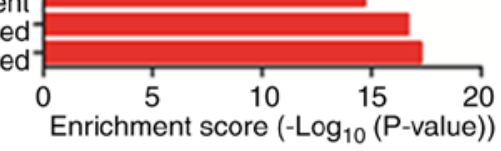

C

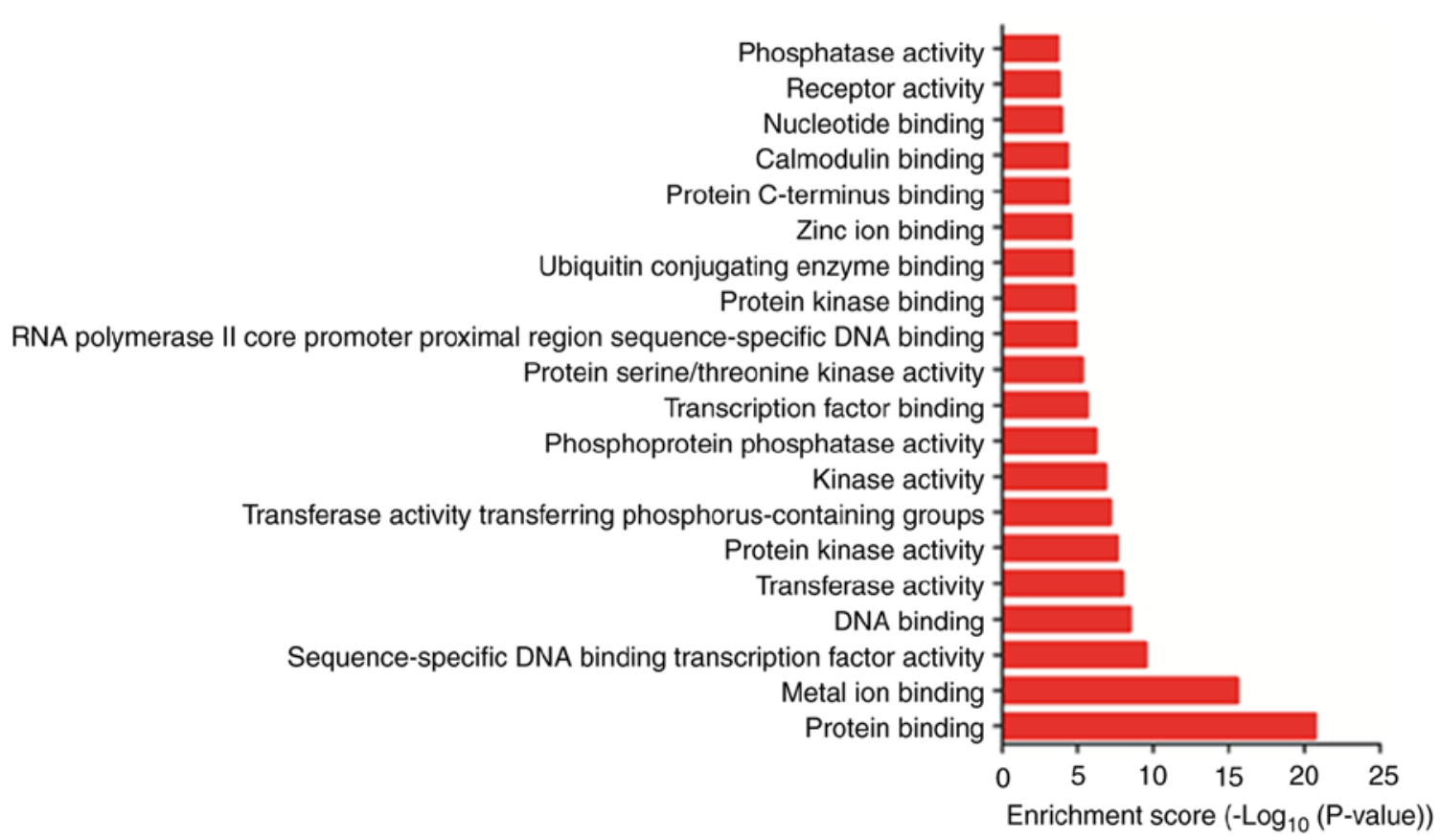

Figure 3. GO term and KEGG pathway analysis of target mRNAs. (A) The number of the predicted mRNA-target genes of the differentially expressed tRFs. Blue, the number of target mRNAs in the miRanda database; Green, the number of target mRNAs in the RNAhybrid database. There were 22,405 shared target mRNAs in miRanda and RNAhybrid. (B) Top $20 \mathrm{GO}$ terms from the target gene enrichment analysis for BP. (C) Top $20 \mathrm{GO}$ terms from the target gene enrichment analysis for MF. The circle size represents the number of genes involved in the pathway. Green, low relative expression; red, high relative expression. GO, Gene ontology; KEGG, Kyoto Encyclopedia of Genes and Genomes; tRFs, tRNA-derived fragments; BP, biological process; MF, molecular function; CC, cellular component.

the occurrence and progression of VVs through the regulation of Wnt, calcium and MAPK signaling pathways.
Several studies have reported that Wnt inhibitory factor 1 can inhibit angiogenesis of human umbilical vein endothelial 
D

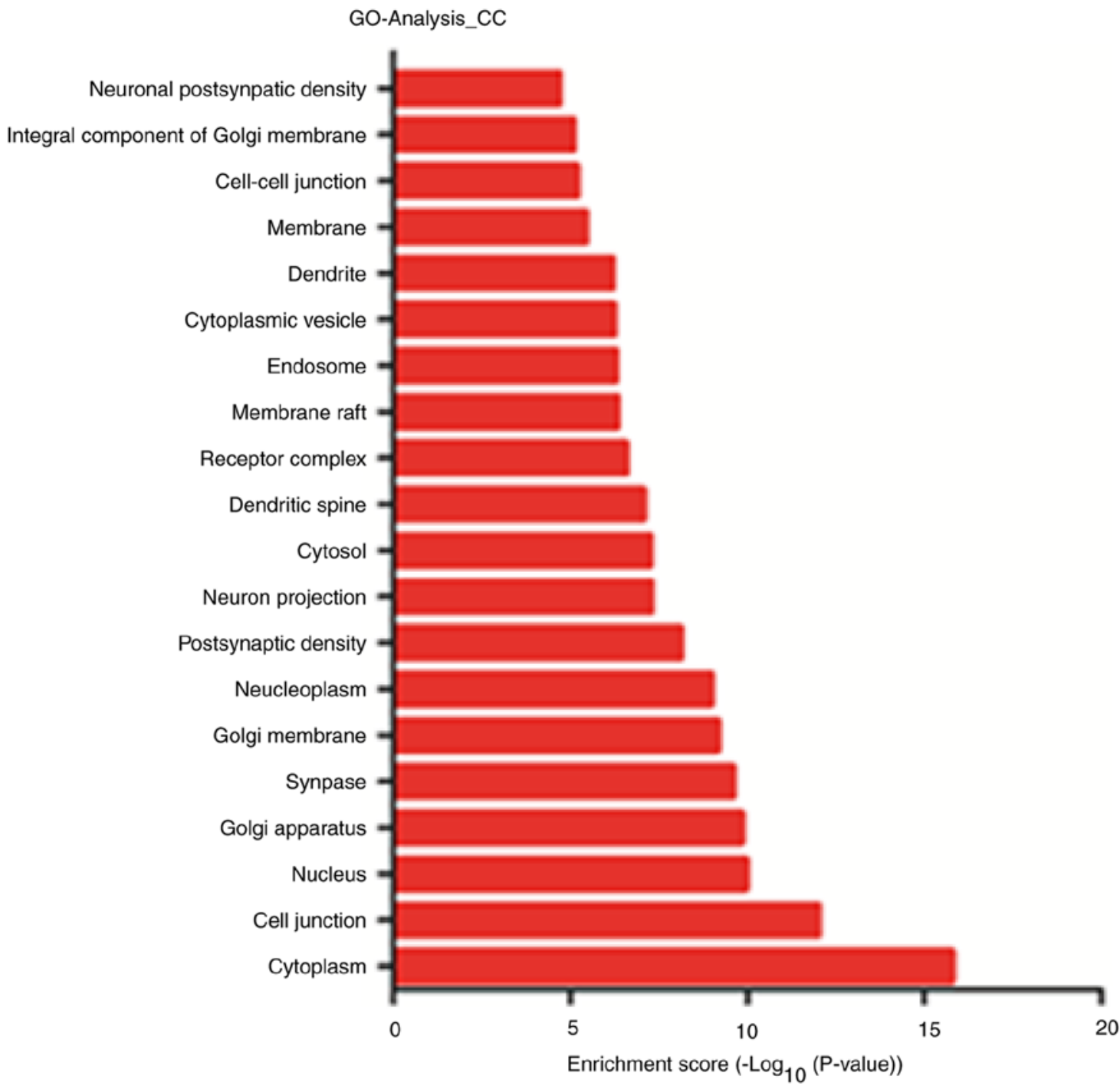

Figure 3. Continued. (D) Top $20 \mathrm{GO}$ terms from the target gene enrichment analysis for CC. The circle size represents the number of genes involved in the pathway. Green, low relative expression; red, high relative expression. GO, Gene ontology; KEGG, Kyoto Encyclopedia of Genes and Genomes; tRFs, tRNA-derived fragments; BP, biological process; MF, molecular function; $\mathrm{CC}$, cellular component.

cells (HUVECs) under hypoxic conditions (37), and activation of the Wnt/ $\beta$-catenin pathway can enhance VEGF-dependent angiogenesis, thus promoting the stability of VEGF-induced new blood vessels in vivo $(30,38)$. In addition, Krüppel-like factor $2 /$ Wnt family member $9 b$ (Klf2/Wnt9b) signaling protects endothelial cells that are sheared by fluid forces and thereby acts directly on the complex cellular processes of heart valve development (39). Previous studies have reported that calcium-independent signaling is responsible for the endothelium-dependent vasodilating activity in HUVECs (40). Tumor-associated calcium signal transducer 2 regulates neovascularization of non-small-cell lung cancer by activating the ERK1/2 signaling pathway (31). In addition, novel calcitonin gene-related peptide/chitosan-strontium-calcium phosphate cement enhances the proliferation of HUVECs (41). Furthermore, increasing extracellular $\mathrm{Ca}^{2+}$ significantly triggers TNF- $\alpha$-induced vascular cell adhesion molecule 1 activation and monocyte adhesion in HUVECs (42). Several reports have revealed that the p38 MAPK inhibitor (CBS3830) restricts vascular re-modeling in arterialized vein grafts (35).
Suppressing the MAPK/NF-kB signaling pathway protects HUVECs from lipopolysaccharide-induced oxidative stress and inflammation (32). Advanced glycation end products (AGE) impair the functions of saphenous vein through the AGE receptor/MAPK signaling pathway in diabetes (43). Studies have also demonstrated that the activation of the MAPK/ERK pathway can enhance the proliferation and migration of HUVECs (44), whereas disrupting MAPK signaling pathway inhibits angiogenesis in HUVECs (45). In addition, CBS3830 restricts vascular remodeling in arterialized vein grafts (35). These reports suggest that Wnt, calcium and MAPK signaling pathways are associated with vascular disease. In the present study, the signaling pathway regulation network revealed that the target genes of the three identified tRFs (tRF-36-F900BY4D84KRIME, tRF-23-87R8WP9IY and tRF-40-86J8WPMN1E8Y7Z2R) were associated with the three signaling pathways. This suggests that alteration in the expression of the three shortlisted tRFs may facilitate the development of novel strategies to manipulate the occurrence and progression of VVs. 


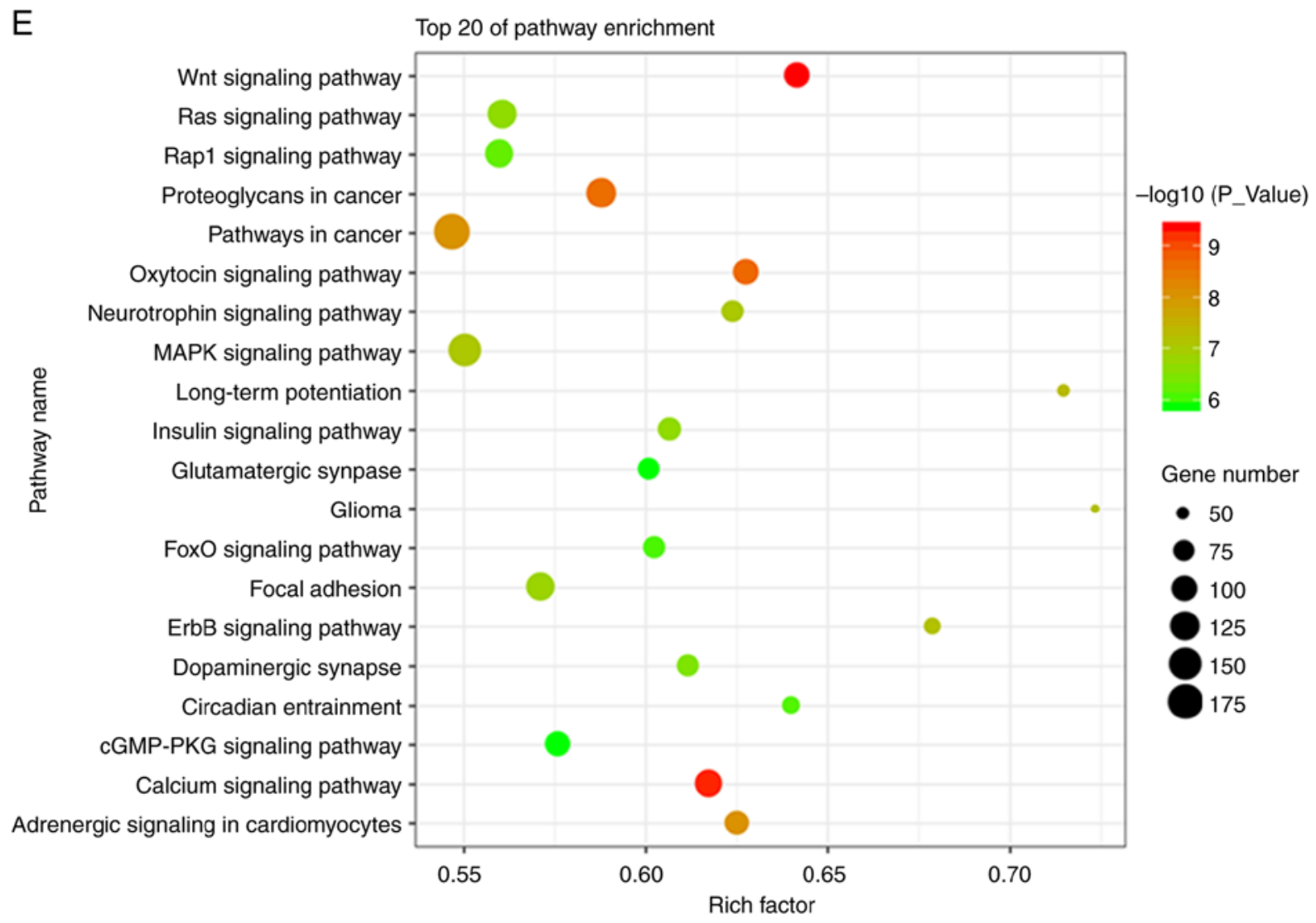

Figure 3. Continued. (E) Top 20 terms from KEGG pathway enrichment analysis of the target genes of the differentially expressed tRFs. The circle size represents the number of genes involved in the pathway. Green, low relative expression; red, high relative expression. GO, Gene ontology; KEGG, Kyoto Encyclopedia of Genes and Genomes; tRFs, tRNA-derived fragments; BP, biological process; MF, molecular function; CC, cellular component.

A

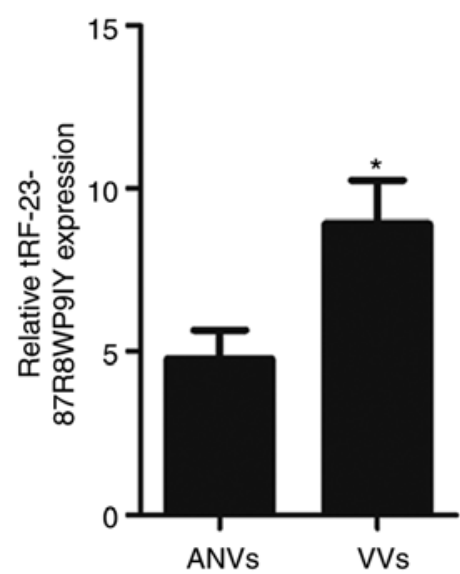

B

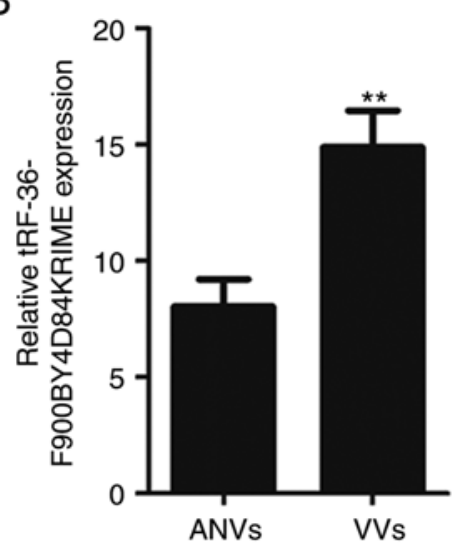

C

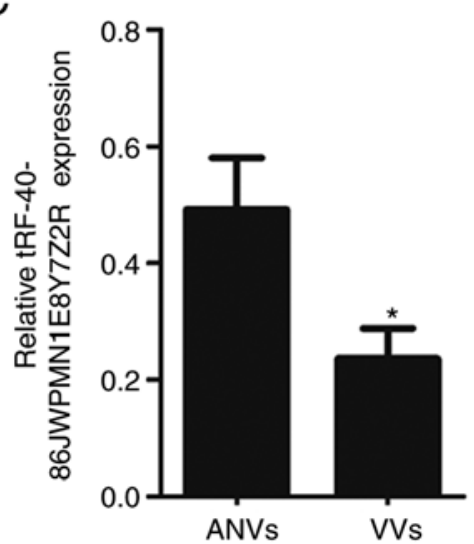

Figure 4. Validation of the DETs in VVs and ANVs. Reverse transcription-quantitative PCR analysis of the expression levels of (A) tRF-23-87R8WP9IY, (B) tRF-36-F900BY4D84KRIME and (C) tRF-40-86J8WPMN1E8Y7Z2R. N=20. Data were analyzed using a paired Student's t-test. ${ }^{*} \mathrm{P}<0.05$ and ${ }^{* *} \mathrm{P}<0.01$ vs. ANVs. tRF, tRNA-derived fragment; DETs, differentially expressed tRFs; VVs, varicose veins; ANVs, adjacent normal veins.

Inflammation has been shown to play an important role in VVs. For instance, polymorphisms of genes associated with inflammation can influence the risk of VVs (46). Lattimer et al (47) found that the concentrations of IL-6, IL-8 and MCP-1 in blood from the site of VVs was increased, which indicated that there may be inflammation in the tissues drained via the VVs. In the present study, several inflammatory factors, including SCD, FKBP5 and ILR6 were found to be regulated by tRF-36-F900BY4D84KRIME, tRF-23-87R8WP9IY and tRF-40-86J8WPMN1E8Y7Z2R, respectively. The high expression of SCD is associated with metabolic diseases, including obesity and insulin resistance, however, SCD is also involved in regulating inflammation and stress in different cell types, such as $\beta$-cells, fat cells, macrophages, endothelial cells and myocytes $(33,34)$. Laminar shear stress plays an important role in vascular homeostasis by increasing the expression of SCD1 in vascular endothelial cells through peroxisome proliferator-activated receptor- $\gamma$ (48). FKBP5 is an FK506 


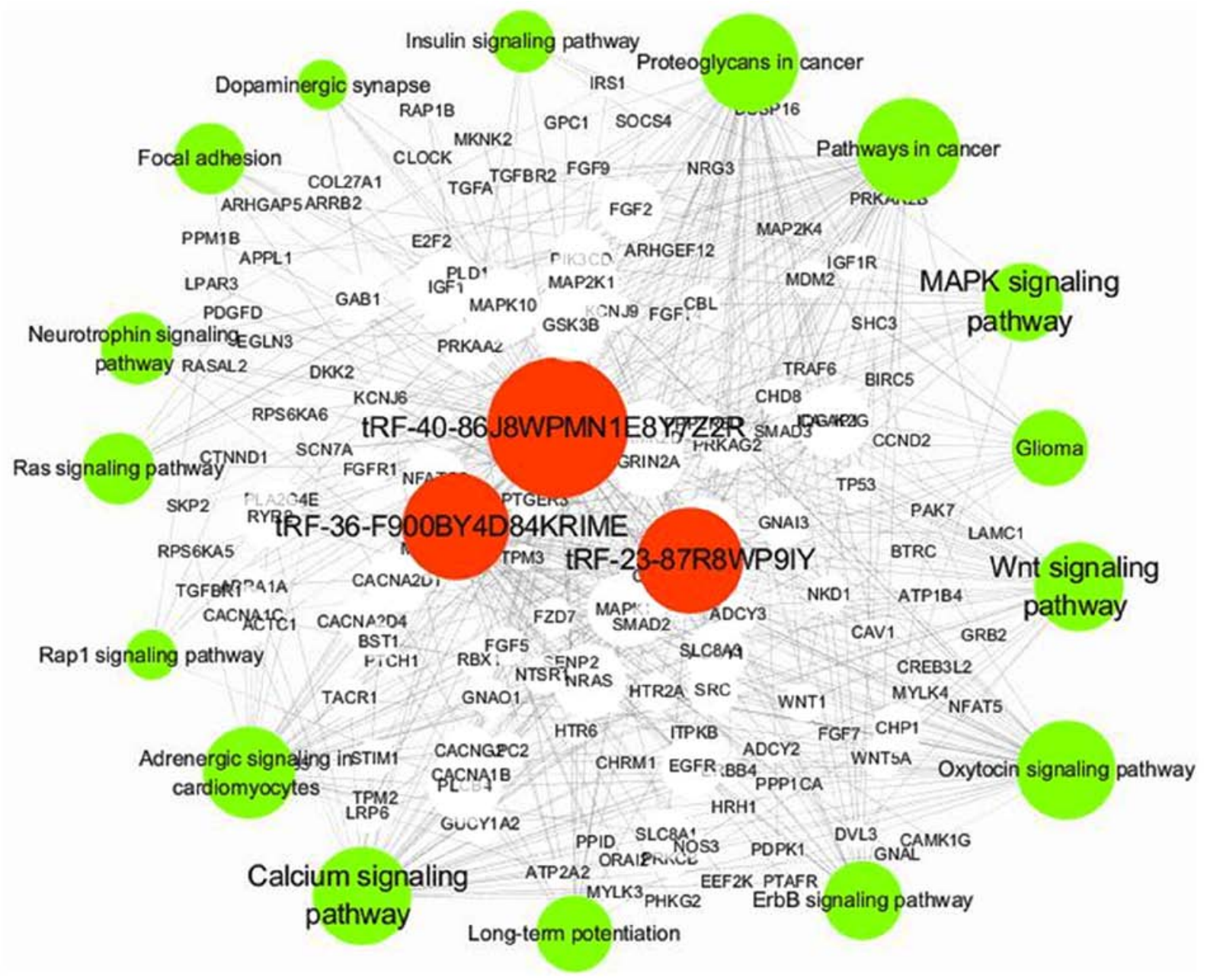

Figure 5. Signaling pathway regulation network of the three validated differentially expressed tRFs. Cytoscape was used to construct the signaling pathway regulation network for the predicted target genes of the three shortlisted tRFs (tRF-36-F900BY4D84KRIME, tRF-23-87R8WP9IY and tRF-40-86J8WPMN1E8Y7Z2R). Red, tRFs; green, signaling pathways; and white, target genes. tRFs, tRNA-derived fragments.

binding protein; FK506 binding proteins are part of the immunophilins family that can bind to immunosuppressive drugs, including rapamycin and FK506 (49). ILR6 is the receptor for IL-6, which is involved in inflammatory reactions (50). The ectopic upregulation of IL6R has been shown to trigger vascular remodeling by regulating inflammatory cell infiltration (51). Therefore, tRFs may serve a regulatory role in VVs by mediating genes involved in inflammation.

In summary,45 DETs were identified in VVs, which included 14 upregulated and 31 downregulated tRFs compared with their expression levels in ANVs. Alterations in the expression of the three shortlisted tRFs, tRF-36-F900BY4D84KRIME, tRF-23-87R8WP9IY and tRF-40-86J8WPMN1E8Y7Z2R, may facilitate in the development of novel strategies for $\mathrm{VV}$ treatment. Further studies will be required to confirm whether tRFs participate in the occurrence and development of VVs through the Wnt, calcium and MAPK signaling pathways. The present study provides a new evidence basis for further investigation of the functional roles of tRFs in VVs.

\section{Acknowledgements}

Not applicable.

\section{Funding}

This study was supported by the Pudong New Area Health and Family Planning Commission Technology Plan (grant no. 2017B-7 to BZ), the Fundamental Research Funds for the Central Universities (grant no. 1507219069 to BZ) and Tongji University Outstanding HR Plan (grant no. 22120180028 to $\mathrm{CY}$ ). The funding bodies had no role in study design, data collection and analysis, decision to publish, or preparation of the manuscript.

\section{Availability of data and materials}

All data generated or analyzed during this study are included in this published article.

\section{Authors' contributions}

$\mathrm{CY}, \mathrm{XW}, \mathrm{YH}, \mathrm{GC}, \mathrm{JG}$ and $\mathrm{BZ}$ conceived and designed the study. CY and XW performed the experiments. YH, GC, HC and JG analyzed and interpreted the data. CY drafted the article. GC, JG and BZ critically revised the manuscript. All authors read and approved the final manuscript. 


\section{Ethics approval and consent to participate}

This study was approved by the Ethics Committee of Shanghai East Hospital (Shanghai, China) and informed consent was obtained from all patients enrolled. All procedures performed in this study involving human participants were in accordance with the ethical standards of the institutional research committee and with the 1964 Helsinki Declaration and its later amendments or comparable ethical standards.

\section{Patient consent for publication}

Not applicable.

\section{Competing interests}

The authors declare that they have no competing interests.

\section{References}

1. Yetkin E, Ileri M and Waltenberger J: Ecchymosis: A novel sign in patients with varicose veins. Clin Hemorheol Microcirc 68: 413-419, 2018.

2. Li X, Jiang XY, Ge J, Wang J, Chen GJ, Xu L, Xie DY, Yuan TY, Zhang DS, Zhang $\mathrm{H}$, et al: Aberrantly expressed lncRNAs in primary varicose great saphenous veins. PLoS One 9: e86156, 2014.

3. Labropoulos N, Tiongson J, Pryor L, Tassiopoulos AK, Kang SS, Ashraf Mansour M and Baker WH: Definition of venous reflux in lower-extremity veins. J Vasc Surg 38: 793-798, 2003.

4. Lim CS, Kiriakidis S, Paleolog EM and Davies AH: Increased activation of the hypoxia-inducible factor pathway in varicose veins. J Vasc Surg 55: 1427-1439, 2012.

5. Jacobs BN, Andraska EA, Obi AT and Wakefield TW: Pathophysiology of varicose veins. J Vasc Surg Venous Lymphat Disord 5: 460-467, 2017.

6. Raffetto JD and Khalil RA: Mechanisms of varicose vein formation: Valve dysfunction and wall dilation. Phlebology 23: 85-98, 2008.

7. Li S, Xu Z and Sheng J: tRNA-Derived small RNA: A novel regulatory small non-coding RNA. Genes (Basel) 9: E246, 2018.

8. Anderson P and Ivanov P: tRNA fragments in human health and disease. FEBS Lett 588: 4297-4304, 2014.

9. Luo S, He F, Luo J, Dou S, Wang Y, Guo A and Lu J: Drosophila tsRNAs preferentially suppress general translation machinery via antisense pairing and participate in cellular starvation response. Nucleic Acids Res 46: 5250-5268, 2018.

10. Goodarzi H, Liu X, Nguyen H C, Zhang S, Fish L and Tavazoie S F: Endogenous tRNA-derived fragments suppress breast cancer progression via YBX1 displacement. Cell 161: 790-802, 2015.

11. Sobala A and Hutvagner G: Small RNAs derived from the 5'end of tRNA can inhibit protein translation in human cells. RNA Biol 10: 553-563, 2013.

12. Ivanov P, Emara MM, Villen J, Gygi SP and Anderson P: Angiogenin-induced tRNA fragments inhibit translation initiation. Mol Cell 43: 613-623, 2011.

13. Couvillion MT, Bounova G, Purdom E, Speed TP and Collins K A Tetrahymena Piwi bound to mature tRNA 3 'fragments activates the exonuclease Xrn2 for RNA processing in the nucleus. Mol Cell 48: 509-520, 2012.

14. Martinez G, Choudury SG and Slotkin RK: tRNA-derived small RNAs target transposable element transcripts. Nucleic Acids Res 45: 5142-5152, 2017.

15. Ruggero K, Guffanti A, Corradin A, Sharma VK, De Bellis G, Corti G, Grassi A, Zanovello P, Bronte V, Ciminale V and D'Agostino DM: Small noncoding RNAs in cells transformed by human T-cell leukemia virus type 1: A role for a tRNA fragment as a primer for reverse transcriptase. J Virol 88: 3612-3622, 2014.

16. Saikia M, Jobava R, Parisien M, Putnam A, Krokowski D, Gao XH, Guan BJ, Yuan Y, Jankowsky E, Feng Z, et al: Angiogenin-cleaved tRNA halves interact with cytochrome $\mathrm{c}$, protecting cells from apoptosis during osmotic stress. Mol Cell Biol 34: 2450-2463, 2014.
17. Wang Z, Xiang L, Shao J and Yuan Z: The 3'CCACCA sequence of tRNAAla(UGC) is the motif that is important in inducing Th1-like immune response, and this motif can be recognized by Toll-like receptor 3. Clin Vaccine Immunol 13: 733-739, 2006.

18. Hafner M, Landthaler M, Burger L, Khorshid M, Hausser J, Berninger P, Rothballer A, Ascano M Jr, Jungkamp AC, Munschauer M, et al: Transcriptome-wide identification of RNA-binding protein and microRNA target sites by PAR-CLIP. Cell 141: 129-141, 2010.

19. Wang Q, Li T, Xu K, Zhang W, Wang X, Quan J, Jin W, Zhang M, Fan G, Wang MB and Shan W: The tRNA-Derived small RNAs regulate gene expression through triggering sequence-specific degradation of target transcripts in the oomycete pathogen phytophthora sojae. Front Plant Sci 7: 1938, 2016.

20. Haussecker D, Huang Y, Lau A, Parameswaran P, Fire AZ and Kay MA: Human tRNA-derived small RNAs in the global regulation of RNA silencing. RNA 16: 673-695, 2010.

21. Pekarsky Y, Balatti V, Palamarchuk A, Rizzotto L, Veneziano D, Nigita G, Rassenti LZ, Pass HI, Kipps TJ, Liu CG and Croce CM: Dysregulation of a family of short noncoding RNAs, tsRNAs, in human cancer. Proc Natl Acad Sci USA 113: 5071-5076, 2016.

22. Balatti V, Pekarsky Y and Croce CM: Role of the tRNA-derived small RNAs in Cancer: New potential biomarkers and target for therapy. Adv Cancer Res 135: 173-187, 2017.

23. Chen Q, Yan M, Cao Z, Li X, Zhang Y, Shi J, Feng GH, Peng H, Zhang X, Zhang Y, et al: Sperm tsRNAs contribute to intergenerational inheritance of an acquired metabolic disorder. Science 351: 397-400, 2016.

24. Garcia-Silva MR, Cabrera-Cabrera F, Güida MC and Cayota A: Novel aspects of tRNA-derived small RNAs with potential impact in infectious diseases. Adv Bioscience Biotechnol 4: $17-25,2013$

25. Zheng LL, Xu WL, Liu S, Sun WJ, Li JH, Wu J, Yang JH and Qu LH: tRF2Cancer: A web server to detect tRNA-derived small RNA fragments (tRFs) and their expression in multiple cancers. Nucleic Acids Res 44: W185-W193, 2016.

26. Shannon P, Markiel A, Ozier O, Baliga NS, Wang JT, Ramage D, Amin N, Schwikowski B and Ideker T: Cytoscape: A software environment for integrated models of biomolecular interaction networks. Genome Res 13: 2498-2504, 2003.

27. Livak KJ and Schmittgen TD: Analysis of relative gene expression data using real-time quantitative PCR and the 2(-Delta Delta C(T)) method. Methods 25: 402-408, 2001.

28. Makki N, Thiel KW and Miller FJ Jr: The epidermal growth factor receptor and its ligands in cardiovascular disease. Int J Mol Sci 14: 20597-20613, 2013.

29. Olsson AK, Dimberg A, Kreuger J and Claesson-Welsh L: VEGF receptor signalling-in control of vascular function. Nat Rev Mol Cell Biol 7: 359-371, 2006.

30. Chen Y, Zhang Y, Deng Q, Shan N, Peng W, Luo X, Zhang H, Baker PN, Tong C and Qi H: Inhibition of wnt inhibitory factor 1 under hypoxic condition in human umbilical vein endothelial cells promoted angiogenesis in vitro. Reprod Sci 23: 1348-1358, 2016.

31. Gosgnach W, Boixel C, Névo N, Poiraud T and Michel JB: Nebivolol induces calcium-independent signaling in endothelial cells by a possible beta-adrenergic pathway. J Cardiovasc Pharmacol 38: 191-199, 2001.

32. Ge JJ, Zhao ZW, Zhou ZC, Wu S, Zhang R, Pan FM and Abendroth DK: p38 MAPK inhibitor, CBS3830 limits vascular remodelling in arterialised vein grafts. Heart Lung Circ 22: 751-758, 2013.

33. Sampath $\mathrm{H}$ and Ntambi JM: The role of stearoyl-CoA desaturase in obesity, insulin resistance, and inflammation. Ann N Y Acad Sci 1243: 47-53, 2011.

34. Liu X, Strable MS and Ntambi JM: Stearoyl CoA desaturase 1: Role in cellular inflammation and stress. Adv Nutr 2: 15-22, 2011.

35. Kim DW, Hwang HS, Kim DS, Sheen SH, Heo DH, Hwang G, Kang SH, Kweon H, Jo YY, Kang SW, et al: Enhancement of anti-inflammatory activity of PEP-1-FK506 binding protein by silk fibroin peptide. J Microbiol Biotechnol 22: 494-500, 2012.

36. Schöniger S, Böttcher D, Theuss T and Schoon HA: Expression of Toll-like receptors 2, 4 and 6 in equine endometrial epithelial cells: A comparative in situ and in vitro study. Res Vet Sci 112: 34-41, 2017.

37. Staniszewska A, Onida S and Davies AH: Compression therapy for uncomplicated varicose veins-Too little for too much? Phlebology 34: 148-150, 2019.

38. Song ZY, Wang F, Cui SX and Qu XJ: Knockdown of CXCR4 Inhibits CXCL-induced angiogenesis in HUVECs through downregulation of the MAPK/ERK and PI3K/AKT and the Wnt/ß-catenin pathways. Cancerinvestigation 36: 10-18, 2018. 
39. Birdsey GM, Shah AV, Dufton N, Reynolds LE, Osuna Almagro L Yang Y, Aspalter IM, Khan ST, Mason JC, Dejana E, et al: The endothelial transcription factor ERG promotes vascular stability and growth through Wnt/ $\beta$-catenin signaling. Dev Cell 32: 82-96, 2015 .

40. Goddard LM, Duchemin AL, Ramalingan H, Wu B, Chen M, Bamezai S, Yang J, Li L, Morley MP, Wang T, et al: Hemodynamic forces sculpt developing heart valves through a KLF2-WNT9B paracrine signaling axis. Dev Cell 43: 274-289.e5, 2017.

41. Guo X, Zhu X, Zhao L, Li X, Cheng D and Feng K: Tumor-associated calcium signal transducer 2 regulates neovascularization of non-small-cell lung cancer via activating ERK1/2 signaling pathway. Tumour Biol 39: 1010428317694324, 2017.

42. Lv T, Liang W, Li L, Cui X, Wei X, Pan H and Li B: Novel calcitonin gene-related peptide/chitosan-strontium-calcium phosphate cement: Enhanced proliferation of human umbilical vein endothelial cells in vitro. J Biomed Mater Res B Appl Biomater 107: 19-28, 2019.

43. Li S, Ning H, Ye Y, Wei W, Guo R, Song Q, Liu L, Liu Y, $\mathrm{Na} \mathrm{L}$, Niu Y, et al: Increasing extracellular $\mathrm{Ca}^{2+}$ sensitizes TNF-alpha-induced vascular cell adhesion molecule-1 (VCAM-1) via a TRPC1/ERK1/2/NFkB-dependent pathway in human vascular endothelial cells. Biochim Biophys Acta Mol Cell Res 1864: 1566-1577, 2017.

44. Xuan H, Yuan W, Chang H, Liu M and Hu F: Anti-inflammatory effects of Chinese propolisinlipopolysaccharide-stimulated human umbilical vein endothelial cells by suppressing autophagy and MAPK/NF- $\mathrm{B}$ signaling pathway. Inflammopharmacology 27 561-571, 2019.

45. Sun Y, Kang L, Li J, Liu H, Wang Y, Wang C and Zou Y: Advanced glycation end products impair the functions of saphenous vein but not thoracic artery smooth muscle cells through RAGE/MAPK signalling pathway in diabetes. J Cell Mol Med 20: 1945-1955, 2016.
46. Liu YW, Zuo PY, Zha XN, Chen XL, Zhang R, He XX and Liu CY: Octacosanol enhances the proliferation and migration of human umbilical vein endothelial cells via activation of the PI3K/Akt and MAPK/Erk pathways. Lipids 50: 241-251, 2015.

47. Lattimer CR, Kalodiki E, Geroulakos G, Hoppensteadt D and Fareed J: Are inflammatory biomarkers increased in varicose vein blood? Clin Appl Thromb Hemost 22: 656-664, 2016.

48. Shadrina A, Tsepilov Y, Smetanina M, Voronina E, Seliverstov E, Ilyukhin E, Kirienko A, Zolotukhin I and Filipenko M: Polymorphisms of genes involved in inflammation and blood vessel development influence the risk of varicose veins. Clin Genet 94: 191-199, 2018.

49. Qin X, Tian J, Zhang P, Fan Y, Chen L, Guan Y, Fu Y, Zhu Y, Chien $S$ and Wang N: Laminar shear stress up-regulates the expression of stearoyl-CoA desaturase-1 in vascular endothelial cells. Cardiovasc Res 74: 506-514, 2007.

50. Van Snick J: Interleukin-6: An overview. Annu Rev Immunol 8: 253-278, 1990

51. Tamura Y, Phan C, Tu L, Le Hiress M, Thuillet R, Jutant EM, Fadel E, Savale L, Huertas A, Humbert M and Guignabert C: Ectopic upregulation of membrane-bound IL6R drives vascular remodeling in pulmonary arterial hypertension. J Clin Invest 128: 1956-1970, 2018

(i) $($ This work is licensed under a Creative Common Attribution-NonCommercial-NoDerivatives 4.0 International (CC BY-NC-ND 4.0) License. 